\title{
An Evolutionary Psychology View of Forgiveness: Individuals, Groups, and Culture
}

Thomas G McCauley $^{1}$, Joseph Billingsley ${ }^{2}$, and Michael E McCullough ${ }^{1}$

${ }^{1}$ University of California, San Diego

${ }^{2}$ Tulane University

Corresponding author: McCullough, Michael E (memccullough@ucsd.edu)

Acknowledgement: The authors gratefully acknowledge support for this work from a grant the John Templeton Foundation (Grant \#29165). 


\begin{abstract}
We review the logic of an evolutionary perspective on forgiveness, highlighting how insight into the likely function of forgiveness--solving adaptive problems related to acquiring and maintaining social relationships--has productively guided research and theory. A combination of experimental, longitudinal, cross-sectional, and cross-cultural evidence supports the claim that victims' perceptions of harmdoers' relationship value and exploitation causally influence whether or not victims forgive harmdoers. We also review the nascent literature on the topic of intergroup forgiveness, and consider how the concepts associated with interpersonal forgiveness, such as apologies, relationship value, and exploitation risk might help us understand forgiveness between groups, cultures, and societies. Finally, we explore the intersection of evolutionary and cultural perspectives on forgiveness, and consider how concepts from these two research traditions might be integrated to help us understand forgiveness even better.
\end{abstract}




\section{Introduction}

Social relationships are fundamental to human life. In even the closest relationships, however, people sometimes harm each other emotionally, socially, materially, and even physically. In the wake of such harms, people often choose to restore relationships rather than abandon them, opting for forgiveness and reconciliation in favor of revenge or avoidance. Forgiveness, the process by which a victim experiences a prosocial change in their motivations towards a transgressor, is crucial for maintaining and repairing relationships, and much empirical attention has been paid to mapping when, why, and how victims forgive their transgressors.

In an influential systematic review, now more than a decade old, Fehr, Gelfand, and Nag [1] summarized what researchers had discovered up to that point about the affective, dispositional, and situational correlates of forgiveness. They concluded that forgiveness was positively associated with empathy, agreeableness, and perspective taking, and negatively associated with anger. They also discovered that people are more likely to forgive when they feel more committed and close to the transgressor, and when they feel more satisfied with the relationship.

In the decade since Fehr, Gelfand, \& Nag's [1] important review, researchers' understanding of forgiveness has improved dramatically, with new insights into many dimensions of forgiveness, including its measurement [2], its personality correlates [3], and its clinical utility [4].

Researchers have also made progress in developing an evolutionary understanding of forgiveness [5-8]. The evolutionary perspective on forgiveness has yielded several unique advances, including the placement of forgiveness within a broader theoretical framework describing the evolutionary logic of helping and harming and an analysis of the information-processing tasks that an evolved system for forgiveness would likely require [9].

Here, we review the recent progress that has emerged from thinking about forgiveness as an evolved feature of human psychology. In addition to reviewing research on interpersonal forgiveness, we apply the evolutionary model of forgiveness to the budding field of intergroup forgiveness and use the evolutionary model to point to nascent findings regarding cultural differences in forgiveness. For all of these topics, we identify high-priority research questions that might merit researchers' attention in the coming years.

\section{The Evolutionary Logic of Forgiveness}

Guided by the principle of natural selection, an evolutionary perspective on psychology rests on the assumption that psychological mechanisms evolve in order to maximize inclusive fitness, a term that designates the additive effects of an individual's direct reproductive success and the reproductive success of the individual's genetic relatives (who, of course, share genes in common in proportion to their degree of relatedness), which raises the reproductive success of

the individual indirectly. On this view, psychological mechanisms adaptively regulate individual 
behavior in response to problems that recurred over evolutionary time [10]. Applying this reasoning to forgiveness, evolution-minded researchers highlight a particular adaptive problemexploitation - that is thought to play a formative role in the emergence of forgiveness psychology [5-7, 9]. Exploitation as conceptualized by these researchers refers to social interaction in which one individual derives fitness benefits ${ }^{1}$ from a second individual at a fitness cost to the latter [5]. Exploitation, whether through the action of lone individuals or of many individuals combining their effort, has persisted in many forms throughout human history and includes such acts as assault, rape, and expropriation of resources, along with a multitude of lesser offenses [11].

When someone has been exploited by another individual, how should the victim best respond? Avoiding the harmdoer is one possibility: If successfully implemented, it could obviate the threat of future exploitation. Avoidance, however, might prove difficult to achieve, especially for individuals who live in small groups. Retaliation, or revenge, is an alternative solution: By inflicting retaliatory costs upon the exploiter, the victim might signal that future exploitationwhether at the hands of the original perpetrator, or by others - would come at too high a price. Though potentially useful as a deterrent, revenge also carries costs of its own. It is a risky strategy because it might invite even further harm, or set in motion a cycle of ever-worsening counter-retaliation. And even if successful, both avoidance and vengeance share another shortcoming: they eliminate or at least diminish a potential flow of fitness benefits that could be realized through future interaction with the exploiter, if more positive relations could be instituted or restored. For harms involving previously close associates, the potential net benefits of cooperation over the long term are vast. Burnette et al. [5] have referred to this disadvantage of avoidance and vengeance as a "second-order adaptive problem" (p. 346), and suggest that the psychological mechanisms that undergird forgiveness evolved in response to it. Forgiveness, on this view, is a solution to the second-order adaptive problems posed by vengeful or avoidant responses to exploitation: It is a shift in interpersonal motivation in the wake of harm, characterized by decreased vengeful or avoidant sentiment, and by increased goodwill, with the ultimate (though not necessarily conscious) purpose of realizing the benefits of future interactions with the transgressor [7, 9].

To take an explicitly evolutionary perspective on forgiveness, a researcher would first want to consider the information-processing features that a mechanism that is well-designed for forgiveness should possess. Recent work has converged on the conclusion that an effective forgiveness mechanism would require at least two crucial internal representations termed relationship value and exploitation risk [5]. Relationship value designates an internal index of the extent to which the victim perceives the transgressor to possess characteristics likely to

\footnotetext{
${ }^{1}$ Fitness benefits refers to the increases in direct or indirect reproductive success that result from the individual's physical and behavioral phenotypes. In the context of exploitation and forgiveness, individuals often win or lose fitness benefits via increases or decreases in their access to valuable resources, additional mating opportunities, or social support of one's children.
} 
increase the victim's welfare [8]: The higher the estimate of relationship value, the stronger the expectation of forgiveness. Exploitation risk designates an internal index of the extent to which the victim perceives the transgressor to possess characteristics likely to reduce the victim's welfare [5]: The higher the estimate of exploitation risk, the weaker the expectation of forgiveness. Following a transgression, the psychological mechanisms that deliver forgiveness should seek out information (e.g., via memory retrieval, direct observation, social inquiry, or other means) that would be useful for estimating both of these quantities. In the case of relationship value, relevant cues likely include genetic relatedness, prior history of productive interaction, similar or complementary interests and background, and membership in the same ethnocultural group. In the case of exploitation risk, relevant cues likely include the egregiousness of the harm, the harmdoer's physical formidability (and other cues to his or her ability to impose costs), and behavioral or communicative signals of benign intent (e.g., apologies, offers of compensation, surrendering one's weapons).

Empirical research has produced results largely in accord with the evolutionary model, especially with respect to perceptions of relationship value. Initially, McCullough et al. [12] conducted a longitudinal study, which revealed that self-reported relationship value predicted a self-report measure of forgiveness, even after accounting for multiple controls. In a correlational design investigating romantic relationships, Burnette et al. ([5], Study 1) likewise found that selfreported relationship value was positively associated with self-reported forgiveness of recalled (though not hypothetical) transgressions. Using three separate samples involving Japanese undergraduates, Japanese adults, and American adults, Smith et al. [13**] replicated the finding that higher relationship value is related to higher self-reported forgiveness. The researchers also found the same positive relationship when using an alternative operationalization of relationship value - namely, the perceived importance of the transgressor to fulfilling one's ongoing goals.

Experimental evidence likewise supports the hypothesized causal role of relationship value. Burnette et al. ([5], Study 2) experimentally manipulated relationship value by asking participants to think of a high vs. low-value individual. In a separate effort, Forster et al. [14] used the Relationship Closeness Induction Task [15], in which participants get to know one another through a guided process of mutual disclosure, to manipulate both relationship value (to create high vs. low-value interaction partners) and transgressor apologies (present vs. absent) in a concurrent double randomization design. In both experiments, greater relationship value increased victim forgiveness of the transgressor.

The evolutionary model predicts that greater exploitation risk will be associated with reduced forgiveness, but research examining exploitation risk is less extensive, and lacking in experimental manipulations. In two studies using recalled and hypothetical transgressions, Burnette et al. [5] found the hypothesized negative correlation between self-reported exploitation risk and self-reported forgiveness (Study 1 and 2), but their attempt to manipulate exploitation 
risk failed (Study 2), so they were unable to estimate its causal effects in a completely unconfounded manner.

Extending the work of ethologists [16-17], evolution-minded researchers have proposed a causal mediation model in which victims' perceptions of relationship value and exploitation risk play critical roles in determining the effectiveness with which transgressor apologies, compensation offers, and other conciliatory gestures elicit forgiveness. According to their model, apologies increase victims' perceptions of the transgressor's relationship value while decreasing their perceptions of the transgressor's exploitation risk. These perceptions, in turn, should increase and decrease forgiveness, respectively. Tabak et al.'s [18] early investigations of this model revealed that the effect of conciliatory gestures on forgiveness was mediated by victims' perceptions of the transgressor's agreeableness - a personality trait construed to index elements of both relationship value and exploitation risk. McCullough et al. [19] examined the mediators more directly in a longitudinal study, and found evidence consistent with the claim that relationship value increased self-reported forgiveness through its intermediate effects on conciliatory gestures; exploitation risk, by contrast, did not appear to decrease forgiveness through such intermediate effects. In their concurrent double randomization experiment, Forster et al. [14**] likewise found that relationship value increased self-reported forgiveness through the intermediate effects of apologies and compensation offers. Forster et al. obtained similar results for a behavioral measure of partner preference, providing strong evidence for the hypothesized causal mechanism. However, Forster and colleagues did not examine exploitation risk as a mediator. Research that is focused on the transgressor's perspective further highlights the intermediate role of relationship value in promoting forgiveness. Using both recalled and hypothetical harms, Ohtsubo and Yagi [20] found that transgressors' perceptions of the victim's relationship value (specifically, the victim's value in helping transgressors reach their goals) predicted how willing transgressors were to make a costly apology to victims, although this finding emerged only when the victim was a friend of the transgressor, not a romantic partner.

Altogether, empirical evidence strongly supports the hypothesis that victims' perceptions of their transgressors' relationship value increase forgiveness, and that apologies and other conciliatory gestures promote forgiveness by altering these perceptions. Some evidence supports a similar role for victim's perceptions of the transgressor's exploitation risk, but further research is needed to evaluate this hypothesis more definitively.

\section{Intergroup Forgiveness and Evolutionary Psychology}

To date, researchers have applied the evolutionary-computational approach to forgiveness almost exclusively to relationships in which one individual has harmed another individual. However, many researchers have focused their efforts over the past decade on understanding how individuals, as members of social groups - ethnic, religious, political - come to forgive other groups. In a recent meta-analytic review Van Tongeren et al. [21] identified several variables that 
predict intergroup forgiveness. Several of those predictors are relevant to our analysis here: People are more willing to forgive offending groups to the extent that (a) the offending group (or representatives speaking on the group's behalf) expressed guilt, made amends, and made themselves seem trustworthy following the harm; (b) the victims identified with a higher-order common identity shared in common with members of the offending group; and (c) the victims interact regularly with members of the other group in daily life.

It is tempting to speculate that factors such as these exert their influence on forgiveness via their intermediate effects on victims' perceptions that the offending group is low in exploitation risk and high in relationship value [22]. However, only one study to our knowledge has directly tested the valuable relationships hypothesis in an intergroup context. Ohtsubo [23*] experimentally manipulated Japanese subjects' perceptions of South Korean people's relationship value by emphasizing South Korea's value as a trade partner. The researchers found that subjects in the high-value condition, in comparison to a control group, were more likely to endorse a peaceful solution to a contentious political issue between Japan and South Korea, relative to subjects in the low-value condition. Although these results suggest that beliefs about a rival group's value as a relationship partner influences individual decisions to forgive groups, it remains to be seen how exploitation risk influences intergroup forgiveness, and whether Ohtsubo et al.'s conclusions generalize to forgiveness between groups that differ on other attributes such as race and religion.

Over the past decade, many researchers have examined the forgiveness-eliciting effects of intergroup apologies, generally understood as public events in which a representative of a social group acknowledges and expresses regret for harms that another group has suffered at the hands of the representative's own group [24]. It is not clear that such apologies reliably promote intergroup forgiveness in the same way they promote interpersonal forgiveness. For example, they do not appear to promote forgiveness among people who identify strongly with the offended group of which they are a member [25]. Wohl et al. [25] provide a number of reasons why intergroup apologies might tend to fail. We suspect, based on evolutionary-computational considerations, that intergroup apologies succeed to the extent that they induce perceptions that members of an offending group retain relationship value and are unlikely to victimize one's own group again in the future.

The notion of intergroup forgiveness introduces other computational complexities that deserve attention. For example, it would be useful to understand how the mind assigns group memberships, assigns responsibility for a harm to a group rather than to one or more concrete individuals, and then assigns responsibility even to members of the group that were not directly involved in perpetrating the harm. How is any group member understood to represent, and potentially speak for, the group itself? Answers to these questions might help provide insight into why many intergroup apologies fail to elicit forgiveness. For instance, perhaps the cognitive link 
connecting the offending group's leader to the group itself is perceived to be weak, thus limiting the extent to which the leader's apology can shift perceptions of the offending group's relationship value. We look forward to future work on intergroup forgiveness that integrates evolutionary-psychology considerations of how we manage to thrive in a world of groups.

\section{Culture and Forgiveness}

Researchers have documented substantial cross-cultural variation in how forgiveness is understood and practiced. Where do these cultural differences come from? Cross-cultural differences in forgiveness have been attributed to variation in the cultural values of individualism and collectivism [26], and other cultural variables might be important as well, but a value-based explanation merely leaves a promissory note as to how those cultural differences emerged in the first place. We think an evolutionary analysis could be helpful here.

Consider a cultural variable called residential mobility, which is often operationalized as the frequency with which people change residence. Numerous studies have shown that residential mobility shapes people's values, along with their beliefs about the importance of those in their groups, networks, and societies [27]. Moreover, mobility is hypothesized to shape the same kinds of social incentives that are central to evolutionary models of forgiveness: The availability of new social partners; the importance of maintaining existing social relationships; and the interdependence between members of dyads, groups, and networks.

A recent study provides initial evidence that residential mobility moderates the effect of relationship value on forgiveness. Komiya et al. [28*] hypothesized that victims from high mobility social environments should perceive their transgressors as less valuable and more easily replaced compared to people from low residential mobility social environments. Consequently, they hypothesized that people from high-mobility backgrounds would require costly apologies (e.g., financial compensation) before forgiving, while low mobility victims would more readily accept a cost-free apology. In three separate experiments, Komiya and colleagues [28] indeed found that people who reported moving more frequently were more likely to forgive after receiving compensation, compared to those who reported moving fewer times; conversely, people who reported moving fewer times were more likely than more frequently movers to forgive after receiving a mere apology (without any compensation). Additionally, Komiya et al. [28] causally manipulated mobility by varying the expected length of subjects' interactions with their partner in a multi-round behavioral economic game. Compensation was most effective in promoting forgiveness among subjects who expected shorter interaction, while apologies sufficed for subjects who anticipated longer interactions.

Komiya et al.'s [28] research is a promising start in integrating cultural and evolutionary considerations, but many questions remain. For instance, what other cultural variables influence 
perceptions of a harmdoer's relationship value? For example, what is the role of the tightnesslooseness dimension (strongly vs. weakly enforced social norms) that Jackson, Choi, and Gelfand [29] proposed as an explanation for cultural differences in revenge? Or consider the approach of Sng et al. [30], who proposed that many cross-cultural differences can be explained by variation in six ecological variables that correspond to adaptive problems that humans universally face: Sex ratio, the genetic relatedness of interaction partners, population density, mortality rate, resource availability, and pathogen prevalence. Approaches such as these deserve additional research attention in the future. Research that shows how cultural variables might exert an influence on forgiveness through their intermediate effects on estimates of relationship value and exploitation risk would be particularly valuable as well.

\section{Conclusion}

Over the past decade, scientific understanding of forgiveness has expanded dramatically, giving us new empirical insights into the causes and effects of forgiveness. An evolutionary approach to forgiveness, we believe, has the potential to integrate all of these findings into an overarching theory that can explain not only how forgiveness works, but also, why humans have the capacity to forgive in the first place [7]. We look forward to additional research on forgiveness from an evolutionary perspective in the decade to come, especially research that can extend our understanding of how forgiveness works between groups and within cultures. 


\section{References and recommended reading}

Papers of particular interest, published within the period of review, have been highlighted as:

- of special interest

- of outstanding interest

[1] Fehr R, Gelfand MJ, Nag M: The road to forgiveness: A meta-analytic synthesis of its situational and dispositional correlates. Psychol. Bull. 2010, 136:894-914. https://doi.org/10.1037/a0019993.

[2] Hodge AS, Captari LE, Mosher DK, Kodali N, Hook JN, Davis DE, Van Tongeren DR: Personality and forgiveness: A meta-analytic review. In Handb. Forgiveness. Edited by Worthington EL, Wade NG. Routledge; 2019:97-106.

[3] McElroy-Heltzel SE, Davis DE, Ordaz AC, Griffin BJ, Hook JN: Measuring forgiveness and self-forgiveness: Descriptions, psychometric support, and recommendations for research and practice. In Handb. Forgiveness. Edited by Worthington EL, Wade NG. Routledge; 2019:74-84.

[4] Webb JR, Toussaint LL: Forgiveness, well-being, and mental health. In Handb. Forgiveness. Edited by Worthington EL, Wade NG. Routledge; 2019:188197.

[5] Burnette JL, McCullough ME, van Tongeren DR, Davis DE: Forgiveness Results From Integrating Information About Relationship Value and Exploitation Risk. Personal. Soc. Psychol. Bull. 2012, 38:345-356.

[6] McCullough M: Beyond revenge: The evolution of the forgiveness instinct. John Wiley \& Sons; 2008.

[7] McCullough ME, Kurzban R, Tabak BA: Cognitive systems for revenge and forgiveness. Behav. Brain Sci. 2013, 36:1-15. https://doi.org/10.1017/S0140525X11002160.

[8] Petersen MB, Sell A, Tooby J, Cosmides L: Evolutionary psychology and criminal justice: A recalibrational theory of punishment and reconciliation. In Human morality and sociality: Evolutionary and comparative perspectives. Edited by Høgh-Olesen H. Palgrave Macmillan; 2010:72-131.

[9] Billingsley J, Burnette JL, \& McCullough, ME: An evolutionary perspective on forgiveness. In Handb. Forgiveness. Edited by Worthington EL, Wade NG. Routledge; 2019:52-62. 
[10] Tooby J, Cosmides L: The psychological foundations of culture. In The Adapted Mind: Evolutionary Psychology and the Generation of Culture. Edited by Barkow JH, Cosmides L, Tooby J, Oxford Univ. Press; 1992:19-136.

[11] Buss DM, Duntley JD: Adaptations for Exploitation. In Gr. Dyn. 2008, 12:53-62. https://doi.org/10.1037/1089-2699.12.1.53.

[12] McCullough ME, Luna LR, Berry JW, Tabak BA, \& Bono G: On the form and function of forgiving: modeling the time-forgiveness relationship and testing the valuable relationships hypothesis. Emotion. 2010, 10:358-376.

[13] Smith A, McCauley TG, Yagi A, Yamaura K, Shimizu H, McCullough ME, Ohtsubo Y: Perceived goal instrumentality is associated with forgiveness: A test of the valuable relationships hypothesis. Evol. Hum. Behav. 2020, 41:58-68. https://doi.org/10.1016/j.evolhumbehav.2019.09.003.

** Provides an empirical test of the evolutionary theory linking relationship value and exploitation risk with forgiveness using both a US and Japanese sample. Three studies that test whether the perceived goal instrumentality of a partner -- a proxy measure of relationship value - influences whether or not a partner is forgiven, and additionally tests whether exploitation risk moderates the effect of goal instrumentality upon forgiveness. The obtained results are consistent with the proposal that relationship value increases forgiveness, but only partially consistent with the premise that a victim's forgiveness is indeed tempered by the likelihood of recidivism by the transgressor.

[14] Forster DE, Billingsley J, Burnette JL, Lieberman D, Ohtsubo Y, McCullough ME: Experimental evidence that apologies promote forgiveness by communicating relationship value. Sci. Rep. 2021, 11:1-14.

** Although past research has shown that apologies promote forgiveness, and that relationship value mediates this effect, no previous research has directly examined the causal link between apologies, relationship value, and forgiveness using an experimental research design that manipulates both the antecedent (apologies) and the mediator (relationship value). The results reported in this paper support the conclusion that apologies obtain their effect by communicating the harmdoer's perceived value of the victim.

[15] Sedikides C, Campbell WK, Reader GD, Elliot AJ: The relationship closeness induction task. Rep. Res. in Soc. Psy. 1991, 23:1-4.

[16] Pokorny JJ, de Waal FBM: Primate questions about the art and science of forgiving. In Handb. of forgiveness. Edited by Worthington EL; 2005:17-32. 
[17] Silk JB: The function of peaceful post-conflict interactions: an alternate view. Nat. conf. Res. Edited by Aureli F, de Waal FBM, Univ. of California Press; 2000:179-181.

[18] Tabak BA, McCullough ME, Luna LR, Bono G, Berry JW: Conciliatory gestures facilitate forgiveness and feelings of friendship by making transgressors appear more agreeable. J. Personality. 2012, 80:503-536.

[19] McCullough ME, Pedersen EJ, Tabak BA, Carter EC: Conciliatory gestures promote forgiveness and reduce anger in humans. Pro. Nat. Aca. Sci. 2014, 111:11211-11216.

[20] Ohtsubo Y, Yagi A: Relationship value promotes costly apology-making: Testing the valuable relationships hypothesis from the perpetrator's perspective. Evol. Hum. Behav. 2015, 36:232-239.

[21] Van Tongeren DR, Burnette JL, O'Boyle E, Worthington Jr EL, Forsyth DR: A meta-analysis of intergroup forgiveness. J. of Positive Psychology. 2014, 9:8195.

[22] Van Tongeren DR, Lindemann R: Intergroup forgiveness. Handb. Forgiveness. Edited by Worthington EL, Wade NG, Routledge/Taylor \& Francis Group; 2020:301-311.

[23] Ohtsubo YL: Relationship value fosters conciliatory attitudes in international conflicts. Peace and Conflict: J. Peace Psych. 2019, 25:259-261.

* This paper provides the first empirical evidence that intergroup forgiveness is informed by the relationship value that groups represent, and reports the results of an experiment that manipulates the perceived value of Korea as a trading partner to Japan in a sample of Japanese subjects. Consistent with an evolutionary perspective on forgiveness, subjects' perceptions of Korea's value positively predicted more conciliatory attitudes. This finding connects existing computational models of forgiveness to what we know about the psychology of groups and coalitions.

[24] Hornsey MJ, Wohl MJ: We are sorry: Intergroup apologies and their tenuous link with intergroup forgiveness. Eur. Rev. of Soc. Psy. 2013, 24:1-31.

[25] Wohl MJ, Hornsey MJ, Philpot CR: A critical review of official public apologies: Aims, pitfalls, and a staircase model of effectiveness. Soc. Issues and Pol. Rev. 2011, 5:70-100. 
[26] Ho MY, Worthington EL: Is the concept of forgiveness universal? A crosscultural perspective comparing western and eastern cultures. Curr. Psyc. 2020, 39:1749-1756.

[27] Choi H, Oishi S: The psychology of residential mobility: a decade of progress. Curr. opinion in psychol. 2020, 32:72-75.

[28] Komiya A, Ozono H, Watabe M, Miyamoto Y, Ohtsubo Y, Oishi S: SocioEcological Hypothesis of Reconciliation: Cultural, Individual, and Situational Variations in Willingness to Accept Apology or Compensation. Front. Psychol. 2020, 11:1761. doi: 10.3389/fpsyg.2020.01761

* Reports three studies suggesting that within-culture variation in the features of people's local environments that are hypothesized to influence the perceived value of social partners is also associated with within-culture variation in forgiveness. The results showed that apologies were more effective for eliciting forgiveness among subjects who moved infrequently (compared to those who did not move frequently). In contrast, compensation is more effective for forgiveness amongst frequent movers (compared to infrequent movers). This paper demonstrates how a socio-ecological approach can explain how cross-cultural differences in forgiveness can be understood in light of evolutionary processes, and how cross-cultural differences in forgiveness might emerge in the first place.

[29] Jackson JC, Choi VK, Gelfand MJ: Revenge: A multilevel review and synthesis. Ann. Rev. Psychol. 2019, 70:319-345.

[30] Sng O, Neuberg SL, Varnum ME, Kenrick DT: The behavioral ecology of cultural psychological variation. Psy. Rev. 2018, 125:714-743. 